\title{
High refractive index of melanin in shiny occipital feathers of a bird of paradise
}

\author{
Doekele G Stavenga $^{1}$, Hein L Leertouwer ${ }^{1}$, Daniel C Osorio ${ }^{2}$ and Bodo D Wilts ${ }^{1, *}$
}

Male Lawes's Parotia, a bird of paradise, use the highly directional reflection of the structurally colored, brilliant-silvery occipital feathers in their courtship display. As in other birds, the structural coloration is produced by ordered melanin pigmentation. The barbules of the Parotia's occipital feathers, with thickness $\sim 3 \mu \mathrm{m}$, contain $6-7$ layers of densely packed melanin rodlets (diameter $\sim 0.25 \mu \mathrm{m}$, length $\sim 2 \mu \mathrm{m}$ ). The effectively $\sim 0.2 \mu \mathrm{m}$ thick melanin layers separated by $\sim 0.2 \mu \mathrm{m}$ thick keratin layers create a multilayer interference reflector. Reflectance measurements yielded peak wavelengths in the near-infrared at $\sim 1.3 \mu \mathrm{m}$, i.e., far outside the visible wavelength range. With the Jamin-Lebedeff interference microscopy method recently developed for pigmented media, we here determined the refractive index of the intact barbules. We thus derived the wavelength dependence of the refractive index of the barbules' melanin to be 1.7-1.8 in the visible wavelength range. Implementing the anatomical and refractive index data in an optical multilayer model, we calculated the barbules' reflectance, transmittance and absorptance spectra, thereby confirming measured spectra.

Light: Science \& Applications (2015) 4, e243; doi:10.1038/lsa.2015.16; published online 30 January 2015

Keywords: bird of paradise; interference reflector; iridescence; Jamin-Lebedeff microscopy; multilayer

\section{INTRODUCTION}

In animal integuments melanins commonly produce dull red, brown and black colors. With nanoscale order, melanin in a matrix of vertebrate keratin or arthropod chitin can produce striking structural colors. ${ }^{1-4}$ The magnificent displays of birds of paradise exemplify how the fine branches of the bird feathers, the barbules, are modified to achieve a diverse range of visual effects through structural coloration. In Lawes's Parotia (Parotia lawesii), the barbules of the males' breast feathers have a boomerang-shaped cross-section, which produces three directional-colored reflectors. ${ }^{5}$ Here we investigate the male Parotia's occipital (or nape) feathers, which produce a shiny, silvery patch (Figure $1 \mathrm{a}$ and $1 \mathrm{~b}$ ). Compared to the breast feathers they are less colorful, but the barbules of the occipital feathers exhibit a mirror-like, directional reflection due to nanostructured melanin. ${ }^{6}$

The uniquely colorful breast feathers allows the breast color to switch sharply between yellow, blue and black as the bird moves, during the ballerina dance, which is performed as part of the courtship display. ${ }^{7-9}$ The shiny occipital feathers have a similar function. Recent behavioral observations on the closely related Wahnes's Parotia demonstrate that the occipital feather reflections are sharply directed to the observing females during part of the courtship performance, presumably to impress a potential mate, viewing from an elevated position on a tree branch. ${ }^{6-10}$

To unravel the optical basis of the shiny occipital reflectors, we investigated the barbule anatomy. This revealed very regularly arranged melanosomes, i.e., small melanin rodlets, arranged in layers. To achieve an in-depth, quantitative understanding of the feathers' reflection characteristics, we measured reflectance and transmittance spectra of the barbules. Additionally, we determined the barbule refractive index by interference microscopy. ${ }^{11-13}$ From the barbule anatomy, we assessed the contribution of melanin to the barbule refractive index and could thus determine the refractive index of melanin as a function of wavelength. By implementing the accumulated data in an optical multilayer model, we could closely reproduce measured angledependent reflectance spectra. The derived melanin data will be significant for detailed, quantitative studies on the coloration of avian plumage and other biological materials that contain melanin.

\section{MATERIALS AND METHODS}

\section{Occipital feathers and anatomy}

Lawes's Parotia occipital feathers (Figure 1a and $1 \mathrm{~b}$ ) were from specimens in the Queensland Museum (Brisbane, Australia) and the Natural History Museum Naturalis (Leiden, The Netherlands). Single feathers were photographed with a Nikon D70 camera (Figure 1c). Feather details were photographed with an Olympus SZX16 stereomicroscope and a Kappa DX40 (Kappa Optronics, Gleichen, Germany) camera (Figure 1d and 1e). Single barbules immersed in a refractive index matching fluid ( $n=1.63$ at $589 \mathrm{~nm}$ ) were photographed with a Zeiss Universal Microscope (Zeiss AG, Oberkochen, Germany) using Nikon Fluor 40/1.30 (Figure 2a) and Zeiss 100/1.25 (Figure 2b and 2c) oil objectives and the Kappa camera. The barbule internal structure was examined with transmission electron microscopy using standard protocols (Figure 2d-2f).

${ }^{1}$ Computational Physics, University of Groningen, 9747 AG, The Netherlands and ${ }^{2}$ School of Life Sciences, University of Sussex, Brighton, BN1 9QG, UK

*Present address: Adolphe Merkle Institute, University of Fribourg, $\mathrm{CH}-1700$ Fribourg, Switzerland

Correspondence: Professor DG Stavenga, Computational Physics, University of Groningen, 9747 AG Groningen, The Netherlands

E-mail: D.G.Stavenga@rug.nl

Received 23 June 2014; revised 19 November 2014; accepted 21 November 2014; accepted article preview online 1 December 2014 

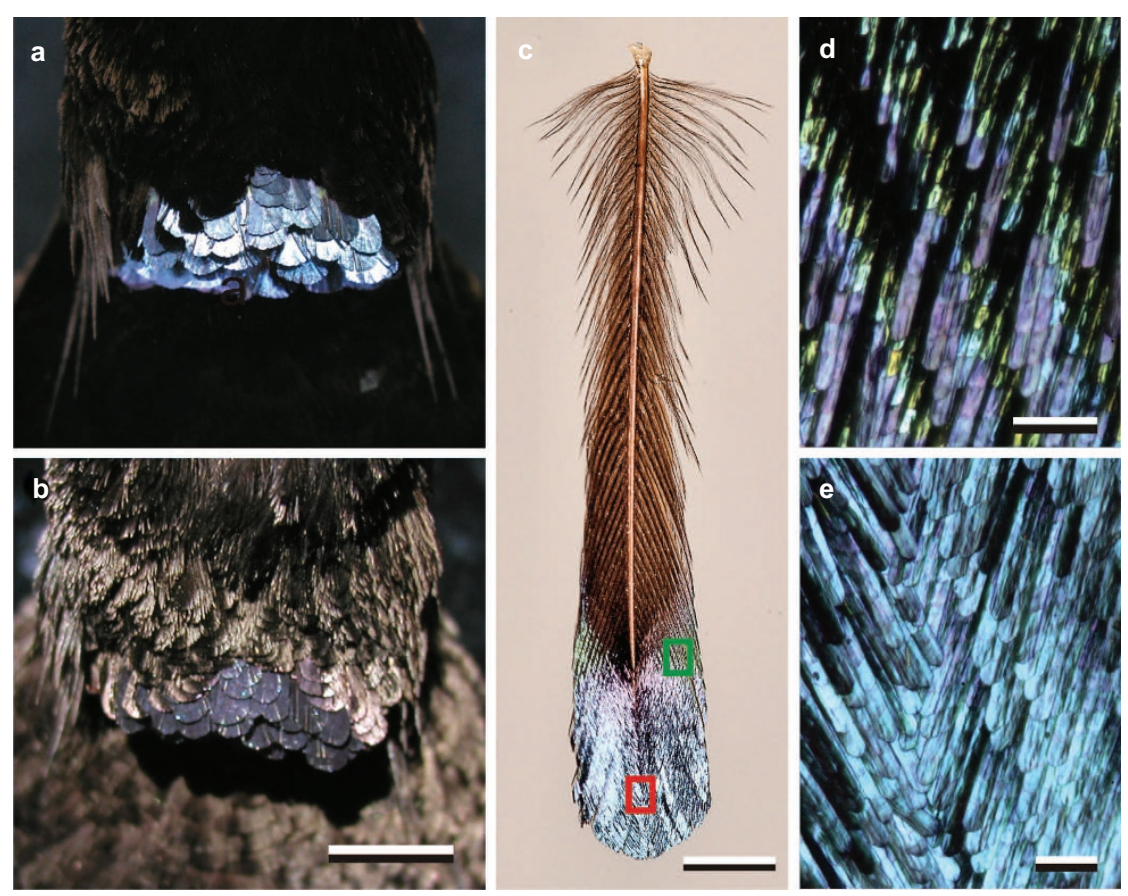

Figure 1 The occipital feathers of Lawes's Parotia. (a) The occipital area illuminated with a narrow-aperture light beam and photographed from the mirror angle to show the reflective occipital feathers. (b) Slight rotation sharply reduces the silvery reflection. (c) A single occipital feather, showing brown proximal barbules, which in situ are covered by other feathers, and silvery-reflective distal barbules, which in situ are exposed. The green and red rectangles correspond to $\mathbf{d}$ and $\mathbf{e}$. (d) The transition area, between the distal and proximal feather parts. The barbule segments here have a variable color, from purple to greenish. (e) With epi-illumination, the distal barbule segments are bluish silvery. Scale bars: a, b 1 cm; c 2 mm; d, e $200 \mu \mathrm{m}$.

\section{Microspectrophotometry}

Reflectance spectra $(R(\lambda), \lambda$ is the light wavelength) of single barbules were measured with a microspectrophotometer: an ultraviolet-visible charge coupled device detector array spectrometer (AvaSpec-2048-2; Avantes, Eerbeek, The Netherlands) attached to a Leitz Ortholux microscope with an Olympus $20 \times$, NA 0.46 objective and a xenon illuminator. The reference was a diffuse white reflectance tile (Avantes WS-2). Transmittance spectra, $T(\lambda)$, were also measured with the microspectrophotometer. The spectra were converted into absorbance spectra with
$D(\lambda)=-\log _{10} T(\lambda)$, which were fitted with the function $D(\lambda)=D_{0} \exp$ $\left(-\lambda / \lambda_{\mathrm{m}}\right)$, because the extinction spectra of the two main melanin types, eumelanin and pheomelanin, approximate exponential functions. ${ }^{13}$

\section{Angle-dependent reflectance measurements}

The angle-dependence of the occipital feather reflectance was measured with a setup consisting of two optical fibers mounted on goniometers that had the same axis of rotation. ${ }^{14}$ The feather's long axis was oriented parallel to the goniometers' plane of rotation (i.e.,
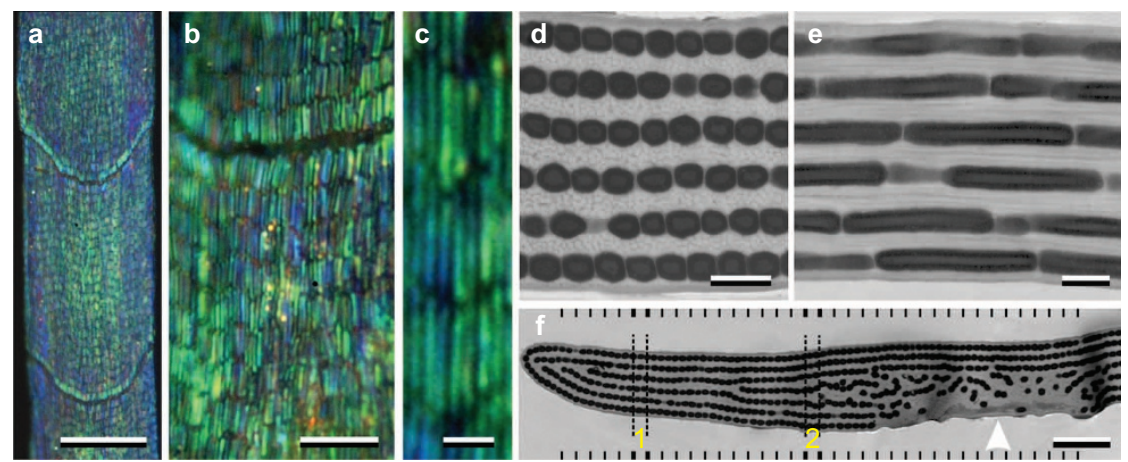

Figure 2 Anatomy of the distal barbules of the occipital feathers. (a) A single barbule immersed in a fluid with refractive index 1.63 (at $589 \mathrm{~nm}$ ) observed with epiillumination light microscopy. The barbule consists of a row of cells. (b) At higher magnification, individual melanin rodlets can be discerned. (c) Close-up view showing the single melanin rodlets, parallel arranged in bands. (d) Transmission electron micrograph of a cross-section, revealing ordered layers of rodlets, diameter $\sim 250 \mathrm{~nm}$, showing a $\sim 60 \mathrm{~nm}$ thick cladding with a slightly higher electron density than the core. (e) Longitudinal section of the rodlets. (f) A cross-section perpendicular to the barbule axis. The layered order of the rodlets is distorted in a central area at the underside (arrowhead). The short vertical bars indicate 36 vertical lanes, $0.5 \mu \mathrm{m}$ wide, for which reflectance, transmittance and absorptance spectra were calculated. For the two lanes flanked by bold bars (numbered 1 and 2 and marked by dotted border lines), calculated refractive index profiles and spectra are given in Figure 4. Scale bars: a $20 \mu \mathrm{m}$; b $5 \mu \mathrm{m} ; \mathbf{c} 1 \mu \mathrm{m} ; \mathbf{d}$, e $0.5 \mu \mathrm{m} ; \mathbf{f} 2 \mu \mathrm{m}$. 
perpendicular to the rotation axis). The first fiber focused light from a xenon lamp onto the feather and was rotated from the normal in steps of $10^{\circ}$. The second fiber collected the reflected light and guided it to a spectrometer: the AvaSpec-2048-2 for the ultraviolet-visible wavelengths and an AvaSpec-NIR256-1.7 for the near-infrared. The resulting signal was always maximal when the second fiber was placed in the mirror-angle position. The second fiber was fitted with an adjustable polarization filter. At each angular position, reflectance spectra were measured for polarized light parallel and perpendicular to the rotation axis, and then were averaged.

\section{Barbule refractive index and feather reflectance modelling}

To determine the refractive index of the melanized barbules, we applied the Jamin-Lebedeff interference microscopy method that we recently developed for measuring the refractive index of absorbing media. ${ }^{12}$ Briefly, we mounted small sections of isolated barbules, immersed in various refractive index fluids (Cargille Labs, Cedar Grove, NJ, USA), on the stage of the Zeiss Universal Microscope set-up for Jamin-Lebedeff interference microscopy, and we thus obtained the mean refractive index of the occipital feather barbules as a function of wavelength, $n_{\mathrm{b}}(\lambda)$; for examples and detailed explanation, see Supplementary Information. Subsequently, we used transmission electron micrographs to determine the contribution of melanin to the refractive index. We therefore divided the transmission electron micrograph of Figure $2 \mathrm{f}$ into 36 lanes, each $0.5 \mu \mathrm{m}$ wide, and recorded the average optical density of a $10 \mathrm{~nm}$ thick cross-section in $3.5 \mu \mathrm{m}$ long lanes. In Figure $2 \mathrm{f}$, dashed boundaries mark two of the lanes, numbered 1 and 2 (\#6 and \#18 of the 36 lanes). We assumed that the areas of the barbule lanes with minimum density, $D_{\min }$, consist of pure keratin, so that the refractive index there was $n_{\mathrm{k}}(\lambda)=1.532+$ $5.89 \times 10^{3} \lambda^{-2}$; $^{11}$ the refractive index outside the barbule was set to 1.0. In addition, we determined the average density of all barbule lanes, $D_{\mathrm{av}}$, and assumed the density difference $D_{\mathrm{av}}-D_{\text {min }}$ to be proportional to the difference of the refractive indices of melanin and keratin, $n_{\mathrm{b}}(\lambda)-n_{\mathrm{k}}(\lambda)$. Scaling the local density accordingly yielded the local refractive index along each lane. We implemented these values in a matrix-based optical multilayer program, ${ }^{14}$ which for each of the 36 lanes produced the wavelength-dependent reflectance, $R$, and transmittance, $T$, as a function of incident angle and polarization. The average of the 36 reflectance spectra was taken to represent the barbule reflectance spectrum. The absorptance was calculated with $A=1-$ $R-T$. The reflectance calculations were also performed for angles of incidence $0^{\circ}, 10^{\circ}, 20^{\circ}, 30^{\circ}, 40^{\circ}$ and $50^{\circ}$ for both TE- and TM- (transverseelectric- and transverse-magnetic-) polarized light, which were then averaged to obtain the reflectance spectra for unpolarized light.

\section{RESULTS AND DISCUSSION}

\section{Feather color and structure}

The male Lawes's Parotia has a silvery-bluish occipital patch (Figure 1a), which is highly reflective in a narrow angle: a slight change of the observation angle causes a large change in the intensity of the reflected light (Figure 1b). The shiny feathers have a reflective structure only on the distal tip. The remainder of the feathers has brown melanin-pigmentation, which is not normally visible, because the feathers overlap each other (Figure 1c). The barbules consist of rows of cells, the color of which can abruptly change from violet to green in the transition area between the brown and silvery regions (Figure 1d). In the main distal part of the feather, the barbules vary little in color (Figure 1e).
The directionality of the reflectivity and the silvery-bluish color suggest a structural origin. Incident light microscopy of the distal barbules in air did not reveal structural details, presumably due to the large refractive index contrast between air and barbule. However, immersion in a high refractive index medium revealed a fine cellular structure in the submicrometer range, with bands of slender, parallel elements, $\sim 2.0 \mu \mathrm{m}$ long and $\sim 0.25 \mu \mathrm{m}$ wide (Figure $2 \mathrm{a}-2 \mathrm{c}$ ). Transmission electron microscopy clarified the inner structure of the barbules. Transverse cross-sections showed that the barbules have a striking internal organisation, with 6-7 layers of closely apposed rodlets (Figure 2d-2f), corresponding to the elements seen with light microscopy (Figure $2 \mathrm{~b}$ and $2 \mathrm{c}$ ). Each rodlet comprises a cladding (thickness $\sim 60 \mathrm{~nm}$ ) surrounding a core with a slightly $(<10 \%)$ lower electron density (Figure $2 \mathrm{~d}$ and $2 \mathrm{e}$ ). The transverse section of Figure $2 \mathrm{f}$ shows that in the central area of the barbule cell the number of layers is reduced and that the parallel layering of the rodlets is locally distorted (Figure 2f, arrowhead). Scanning electron microscopy demonstrated that the barbule cells' upper surface is smooth, but at the underside, a valley exists in the center of each cell (not shown). The valley and the layer distortion are most probably the remains of the nucleus of the dead cell. ${ }^{1}$

\section{Barbule pigmentation}

The multilayer structure of the barbule's interior is clearly the basis of the highly directional reflectivity of the occipital feathers. ${ }^{6}$ In order to understand the barbule optics in further detail, we wanted to know the material properties of the rodlets and its surroundings, and therefore we performed microspectrophotometry on single barbule cells. The reflectance spectra of small areas of different barbule cells (Figure 3a, inset right) were broad-band and peaked in the blue-violet, and the far-red wavelength range featured striking oscillations with amplitude increasing with wavelength (Figure 3a).

When observing the barbules with epi-illumination from the underside, a central contrasting area is seen in each cell (Figure 3a, inset left). When the same barbule is observed in transmitted light (Figure 3b, inset), the cell centers are marked by a light-brown color within the generally brown barbule. The brown color strongly suggests the presence of melanin pigment, and the central light-brown color indicates a lower melanin concentration in the cell centers. This can be immediately related to the depressed central area of Figure $2 \mathrm{f}$, with fewer layers of rodlets.

Transmittance spectra measured of the main barbule cell area and the cell center had the same shape, but they differed in amplitude (Figure $3 \mathrm{~b}$ ). The measured transmittance spectra converted to absorbance spectra approximated an exponential function, $D=D_{0} \exp (-\lambda /$ $\lambda_{\mathrm{m}}$ ), with $\lambda_{\mathrm{m}}=155 \pm 8 \mathrm{~nm}$. The absorbance spectra of pheomelanin and eumelanin, which often coexist in bird feathers, ${ }^{15}$ are described by exponential functions with $\lambda_{\mathrm{m}}=115 \mathrm{~nm}$ and $\lambda_{\mathrm{m}}=175 \mathrm{~nm}$, respectively, ${ }^{13}$ suggesting that the pigment of the bird of paradise is predominantly eumelanin. We conclude that the barbules consist of melanin rodlets embedded in the main component of bird feathers, keratin.

\section{Barbule and melanin refractive index}

Melanin has a higher refractive index than keratin, ${ }^{1}$ and the arrangement of the melanin rodlets in layers will cause the barbules to act as an optical multilayer. For a quantitative understanding of the measured reflectance and transmittance spectra, the refractive index of the constituent layers has to be known. We first determined the refractive index as a function of wavelength of both the main barbule area and the cell center by applying Jamin-Lebedeff interference microscopy ${ }^{12}$ 


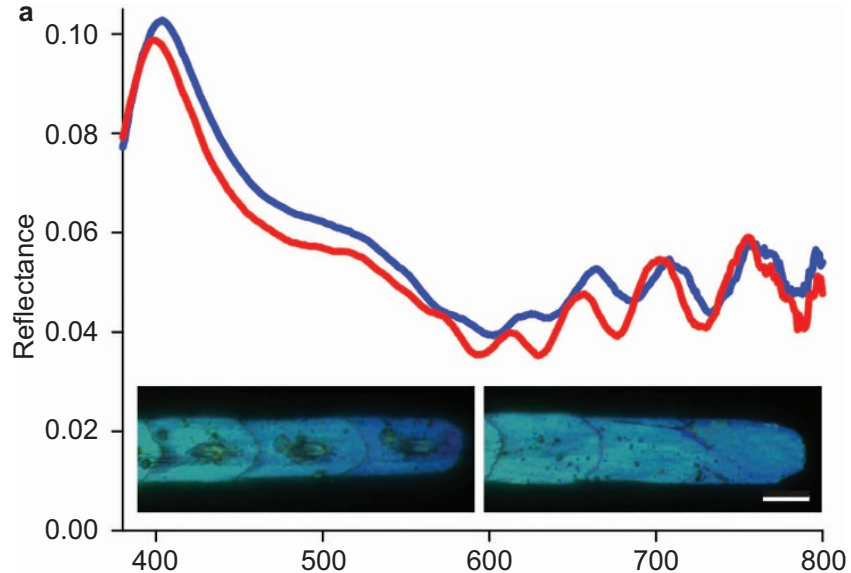

b
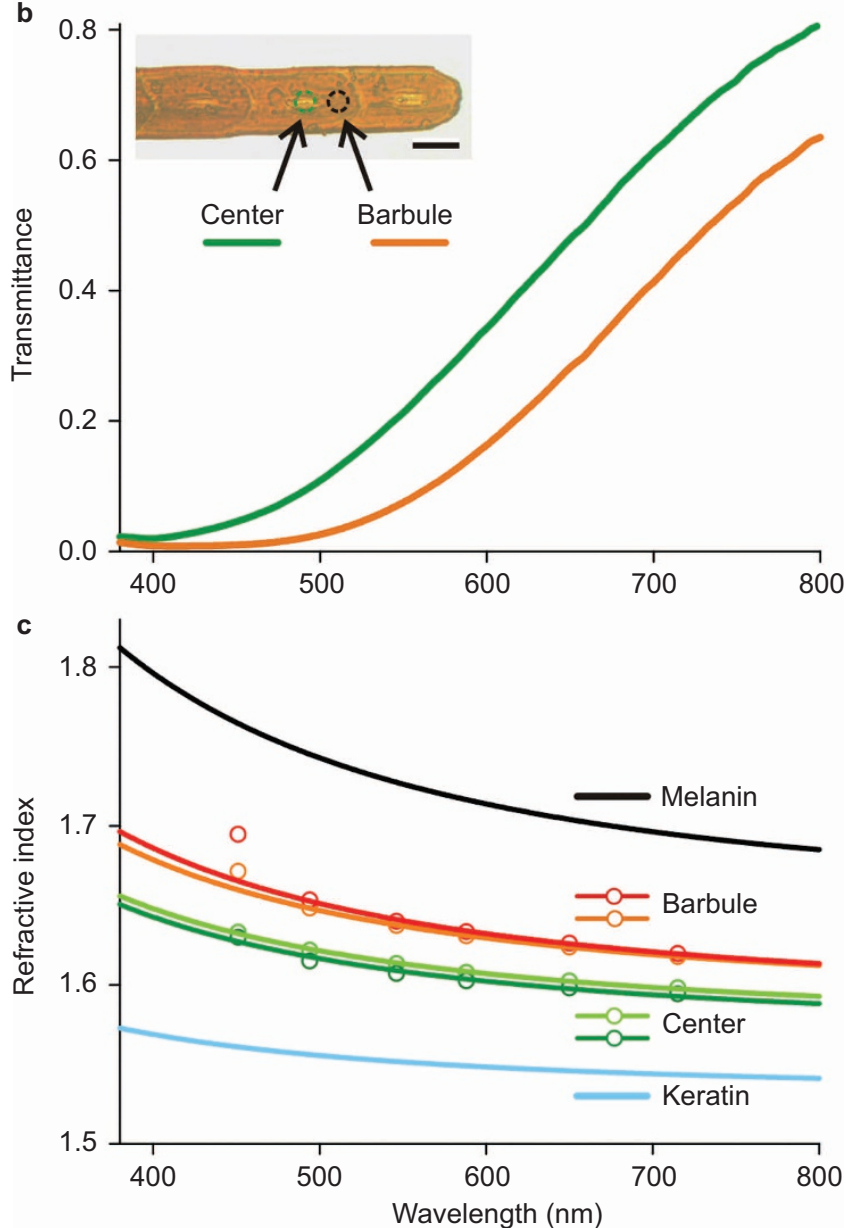

Figure 3 Reflectance, transmittance and refractive index of occipital feather barbules. (a) Reflectance spectra measured from small barbule areas $(\sim 5 \mu \mathrm{m}$ diameter, upperside) showing oscillations reminiscent of thin film interference. Insets: a barbule observed with epi-illumination from the underside (left) and upperside (right; scale bar: $20 \mu \mathrm{m}$ ). (b) Transmittance spectra measured from the center of a barbule cell and from an area outside the center. Inset: the same barbule as that in a observed with transmitted light (scale bar: $20 \mu \mathrm{m}$ ). (c) Refractive index of barbule and center areas as a function of wavelength, derived from Jamin-Lebedeff interference microscopy, with Cauchy function fits, together with the refractive index of bird keratin ${ }^{11}$ and the derived melanin refractive index.
(Figure 3c; see also Supplementary Figs. S1 and S2). The refractive index spectra were well described by the Cauchy equation: $n(\lambda)=$ $A+B / \lambda^{2}$, where for the main barbule area $A_{\mathrm{b}}=1.590 \pm 0.001$ and $B_{\mathrm{b}}=(1.48 \pm 0.07) \times 10^{4} \mathrm{~nm}^{2}$, and for the center $A_{\mathrm{c}}=1.572 \pm 0.002$ and $B_{\mathrm{c}}=(1.17 \pm 0.01) \times 10^{4} \mathrm{~nm}^{2}$ (Figure $3 \mathrm{c}$ ).

The derived refractive index spectra are weighted sums of the refractive indices of the keratin and melanin constituents. For the keratin of white goose feathers, we found Cauchy parameters $A_{\mathrm{k}}=$ $1.532 \pm 0.001$ and $B_{\mathrm{k}}=(5.89 \pm 0.02) \times 10^{3} \mathrm{~nm}^{2}$ (Figure 3c). ${ }^{11}$ Assuming that the keratins of bird of paradise and goose feathers are alike, this means for the main barbule area a difference in Cauchy parameters with those of keratin $\Delta A_{\mathrm{b}}=A_{\mathrm{b}}-A_{\mathrm{k}}=0.058 \pm 0.002$ and $\Delta B_{\mathrm{b}}=$ $B_{\mathrm{b}}-B_{\mathrm{k}}=(0.89 \pm 0.09) \times 10^{4} \mathrm{~nm}^{2}$, while for the center area, $\Delta A_{\mathrm{c}}=$ $A_{\mathrm{c}}-A_{\mathrm{k}}=0.040 \pm 0.003$ and $\Delta B_{\mathrm{c}}=B_{\mathrm{c}}-B_{\mathrm{k}}=(0.58 \pm 0.03) \times 10^{4} \mathrm{~nm}^{2}$. Because $\Delta A_{\mathrm{c}} / \Delta A_{\mathrm{b}} \approx \Delta B_{\mathrm{c}} / \Delta B_{\mathrm{b}} \approx 2 / 3$, the increase in the effective refractive index in the center with respect to keratin is about $2 / 3$ of the refractive index increase in the main barbule area. This agrees with the anatomical data, showing an approximately $2 / 3$ concentration of electron dense material in the center area compared to that in the surrounding area (Figure $2 \mathrm{f}$ ).

The barbule refractive index depends on the relative amounts of keratin and melanin. If we assume that the barbules contain an equal amount of keratin and melanin (Figure 2), with refractive indices $n_{\mathrm{k}}$ and $n_{\mathrm{m}}$, respectively, the barbule refractive index is $n_{\mathrm{b}}=\left(n_{\mathrm{k}}+n_{\mathrm{m}}\right) / 2$, or $n_{\mathrm{m}}=2 n_{\mathrm{b}}-n_{\mathrm{k}}$. We thus derive that the refractive index of melanin is described by Cauchy parameters $A_{\mathrm{m}}=1.648 \pm 0.003$ and $B_{\mathrm{m}}=$ $(2.37 \pm 0.14) \times 10^{4} \mathrm{~nm}^{2}$. This concerns the real part of the refractive index, of course. The imaginary part can be derived similarly. Using the measured transmittance spectra (Figure 3b), the imaginary part of the refractive index of the melanin in the visible wavelength range appears to be well fitted by the exponential function $k_{\mathrm{m}}(\lambda)=a_{\mathrm{m}}$ $\exp \left(-\lambda / b_{\mathrm{m}}\right)$, with $a_{\mathrm{m}}=0.56 \pm 0.01$ and $b_{\mathrm{m}}=270 \pm 5 \mathrm{~nm}$.

The refractive index of melanin in feathers has been a matter of conjecture for several decades. For the pigeon Columba trocaz, whose barbule cells contain melanin granules and air spaces in a keratin matrix, application of a series of immersion fluids led Schmidt ${ }^{1}$ to conclude that the granule refractive index exceeds 1.739 ( $n_{\mathrm{D}}$ of methylene iodide), that it approximates 1.76-1.77 (ruby and sapphire) and is less than 2.42 (diamond). We found here that the refractive index of the melanin in the visible wavelength range is 1.7-1.8 (Figure 3c), which is in close agreement with Schmidt. ${ }^{1}$ This value is however distinctly lower than 2.0, the value commonly used in animal coloration studies, although without experimental justification (e.g. Refs. 2 and 16-18).

\section{Multilayer modeling}

The refractive index data of keratin and melanin can be used to calculate the reflection and transmission properties of the barbules by optical multilayer modelling. The melanin rodlets do not create simple planoparallel layers, however. The electron micrographs of Figure $2 \mathrm{~d}-$ $2 \mathrm{f}$ rather suggest that the barbules have a gradient refractive index. To quantitatively assess the refractive index gradient, we divided the barbule cross-section of Figure $2 \mathrm{f}$ into 36 adjacent lanes, each $0.5 \mu \mathrm{m}$ wide. We assumed each lane to be a stack of $10 \mathrm{~nm}$ thick layers and determined the average electron density in each layer. We then took the lowest electron density, $D_{\min }$, and the average density of all barbule lanes, $D_{\mathrm{av}}$, and assumed the difference, $D_{\mathrm{av}}-D_{\min }$, to be proportional to the difference of the derived refractive index value of melanin with the refractive index of keratin, $n_{\mathrm{b}}(\lambda)-n_{\mathrm{k}}(\lambda)$. By then scaling the local density in each of the $10 \mathrm{~nm}$ layers in all lanes, we obtained the 
refractive index profile of all lanes. Figure 4a shows the results, at $\lambda=500 \mathrm{~nm}$, for the two lanes marked in Figure $2 \mathrm{f}$. We have to note here that we derived above that the refractive index of melanin at $500 \mathrm{~nm}$ is $1.74 \pm 0.01$, while the profiles of Figure $2 \mathrm{f}$ show that the scaling procedure can produce locally higher values. This is clearly due to the inhomogeneity of the melanin layers. In fact, because the values represent the average refractive index of a $10 \mathrm{~nm}$ thick and $0.5 \mu \mathrm{m}$ wide layer, refractive index values $>1.8$ will be reached locally. Yet, the average values will hold when considering the reflection and transmission of the propagating light flux.

The first step in obtaining the feather's reflectance spectrum was to calculate for each lane the reflectance, transmittance, and absorptance spectra by feeding the lane's refractive index profile into a multilayer model. ${ }^{14}$ The two barbule lanes of Figure 4 a yielded for normal illumination the spectra shown in Figure $4 \mathrm{~b}$. The calculated reflectance spectra always peaked in the infrared and furthermore showed clear oscillations in the far-red wavelength range (Figure 3a). The peak in the infrared is due to the multilayered interference reflector inside the barbule, but the oscillations arise because the barbules simultaneously
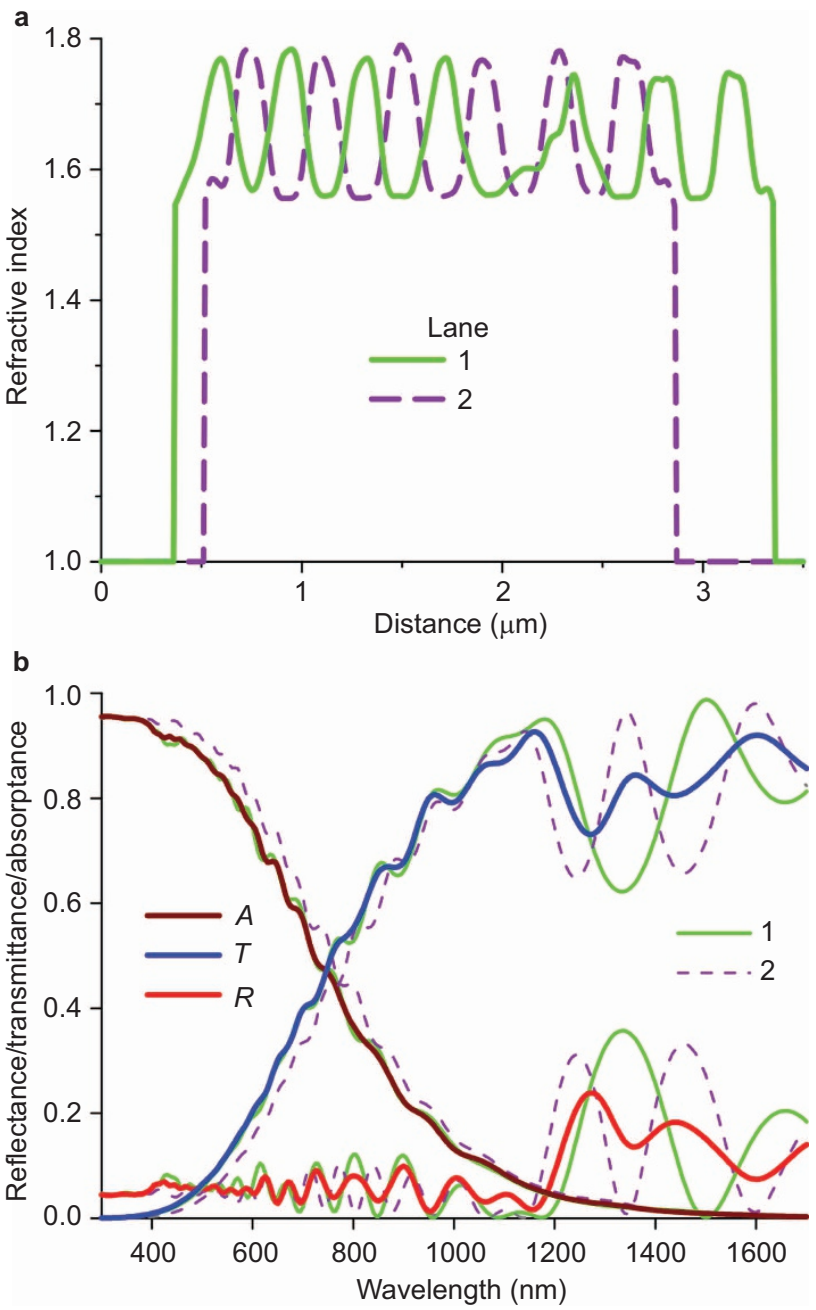

Figure 4 Refractive index profiles and their spectral consequences. (a) The real part of the refractive index at $500 \mathrm{~nm}$ for the two $0.5 \mu \mathrm{m}$ wide lanes numbered 1 and 2 in Figure 2f. (b) Absorptance $(A)$, transmittance $(T)$ and reflectance $(R)$ spectra for normally incident light, calculated with a multilayer model for lanes 1 and 2 and the averaged spectra (brown, blue and red curves, respectively). act as a thin film with thickness $\sim 3 \mu \mathrm{m}$ (Supplementary Fig. S3). ${ }^{19}$ The transmittance spectra (Figure $4 \mathrm{~b}$ ) showed a steep increase with increasing wavelength, clearly due to the melanin, which absorbs progressively less with increasing wavelength (Figure $3 \mathrm{~b}$ ).

We averaged the reflectance spectra for all 36 lanes, yielding a much smoother spectrum than those of Figure $4 \mathrm{~b}$ (Figure $5 \mathrm{a}, 0^{\circ}$ ). The dependence of the reflectance on the angle of light incidence can be easily obtained by multilayer modeling, and thus, we repeated the calculations for a series of angles of light incidence, changed in steps of $10^{\circ}$ (Figure $5 \mathrm{a}$ ). Because multilayer reflections depend on the polarization, we calculated the spectra for both TE- and TM-polarized light (Supplementary Fig. S4a and S4c). By averaging the TE- and TMspectra for each angle of light incidence we obtained the reflectance spectra for unpolarized light (Figure 5a). As expected for a multilayer, with increasing angle of incidence, the infrared peak shifted to shorter wavelengths (Supplementary Fig. S5).

To verify the validity of the calculations, we measured the angledependent reflectance of the occipital feather tip in both the visible and infrared wavelength range with a setup consisting of two rotatable optical fibers. The illumination spot, with a spot diameter of $\sim 4 \mathrm{~mm}$, approximately covered the entire shiny part of the feather. The illumination fiber angle was varied in $10^{\circ}$ steps and at each step, the detection fiber was moved until a maximal signal was obtained. This
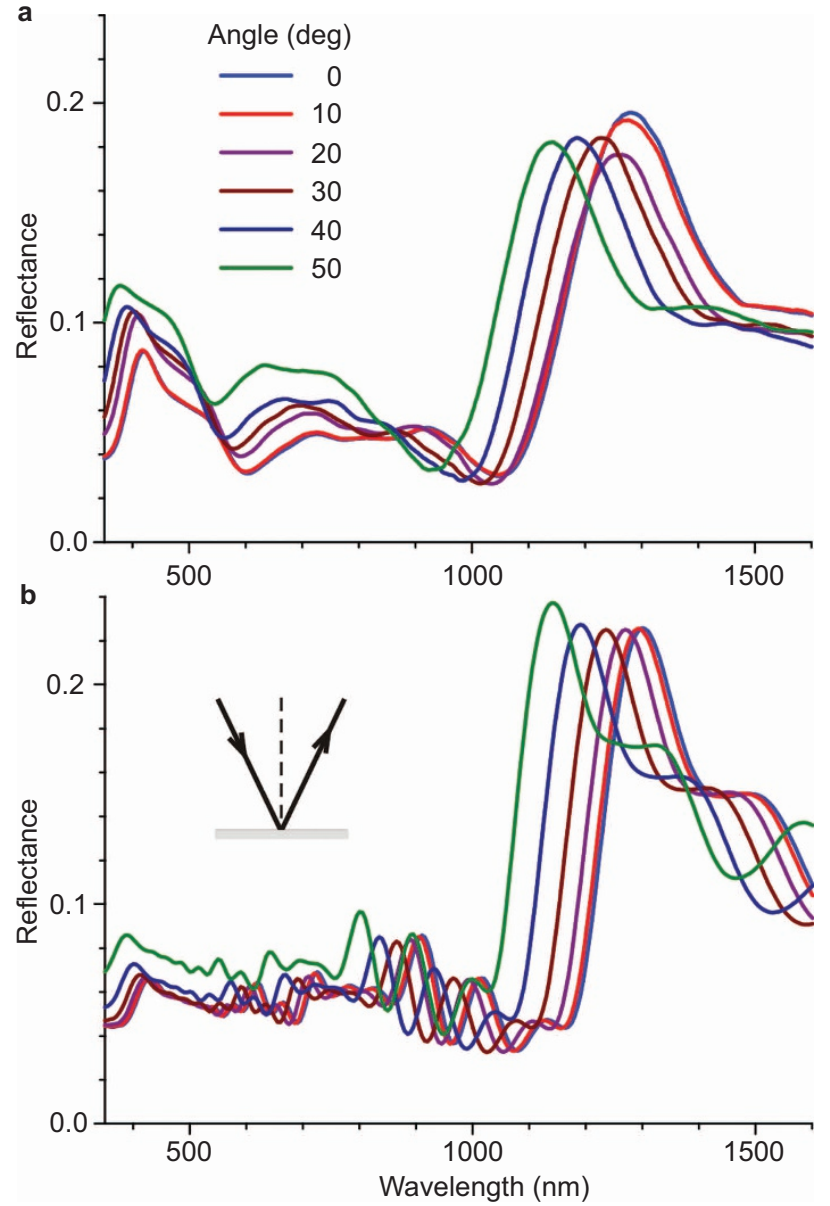

Figure 5 Angle-dependent reflectance spectra. (a) Averaged spectra calculated for 36 lanes of the transmission electron micrograph of Figure 2f, for angles of incidence $0^{\circ}-50^{\circ}$. (b) Reflectance spectra measured for each angle of incidence at the mirror angle. 
position was always identical to the mirror angle (Figure $5 \mathrm{~b}$, inset). The measurements were done for both TE- and TM-polarized light and these spectra were then again averaged (Figure 5b). The experimental and calculated spectra were not identical, but the overall behavior was clearly very similar (Figure $5 \mathrm{a}$ and $5 \mathrm{~b}$ ).

A perfect correspondence cannot be expected, because the calculations were for a cross-section of only part of a single barbule cell, while the experimental reflectance spectra were from an intact feather, which contains numerous barbules, and their cells were certainly not all identical. The spectral calculations were performed for $0.5 \mu \mathrm{m}$ wide lanes, because even within one and the same barbule cell the multilayering is far from ideal (Figure 2f). The averaged reflectance spectra resulting from the set of 36 parallel lanes nevertheless showed a close correspondence with the experimental spectra concerning the peak wavelength in the infrared and a similar hypsochromic spectral shift with increasing angle of light incidence, indicating the validity of the heuristic approach. In fact, finite-difference time-domain modeling ${ }^{6}$ yielded reflectance spectra matching those of the multilayer modeling (compare Figure 2 of Ref. 6 and Supplementary Fig. S3). Moreover, the present reflectance spectra measured on the multi-colored breast feathers of Lawes's Parotia ${ }^{5}$ were straightforwardly produced by a finitedifference time-domain model based on the quite different feather anatomy while using the melanin refractive index derived here. ${ }^{6}$

\section{CONCLUSIONS}

Many birds have iridescent feathers due to melanin rodlets arranged in layers. The refractive index value of melanin derived here by applying Jamin-Lebedeff interference microscopy decreases in the visible wavelength range $(400-600 \mathrm{~nm})$ gradually from $\sim 1.8$ to $\sim 1.7$, and thus shows normal dispersion. A constant refractive index value 2.0 , which is commonly used in bird coloration studies, is clearly much too high. Moreover, the absorption of melanin is usually neglected in modeling studies, that is, the imaginary part of the melanin refractive index is assumed to be constant. Here we have found that the strongly wavelength-dependent melanin absorption distinctly influences the optical properties of bird feathers. The combined modeling and reflectance measurements of the present study confirm the measured melanin refractive index spectrum. The obtained melanin data will be useful for modeling the feather reflectance properties of birds with structural coloration due to melanin multilayers.

\section{ACKNOWLEDGEMENTS}

We thank NJ Marshall for the photographs of Lawes's Parotia, J Thorpe for the transmission electron microscopy and A Vey for comments. This study was financially supported by AFOSR/EOARD (grant FA8655-08-13012).

1 Schmidt WJ. Wie entstehen die Schillerfarben der Federn? Naturwiss 1952; 39: 313_ 318.

2 Durrer H. Schillerfarben der Vogelfeder als Evolutionsproblem. Denkschr Schweiz Naturforsch Ges 1977: 91: 1-127.

3 Prum RO. Anatomy, physics, and evolution of avian structural colors. In: Hill GE, McGraw KJ, editors. Bird Coloration. Vol. I, Mechanisms and Measurements. Cambridge, MA: Harvard University Press; 2006. p295-353.

4 Nakamura E, Yoshioka S, Kinoshita S. Structural color of rock dove's neck feather. J Phys Soc Jpn 2008; 77: 124801.

5 Stavenga DG, Leertouwer HL, Marshall NJ, Osorio D. Dramatic colour changes in a bird of paradise caused by uniquely structured breast feather barbules. Proc R Soc B2011; 278: 2098-2104

6 Wilts BD, Michielsen K, De Raedt H, Stavenga DG. Sparkling feather reflections of a bird-of-paradise explained by finite-difference time-domain modeling. Proc Natl Acad Sci USA 2014; 111: 4363-4368.

7 Pruett-Jones SG, Pruett-Jones MA. Sexual selection through female choice in Lawes' Parotia, a lek-mating bird of paradise. Evolution 1990; 44: 486-501.

8 Frith CB, Beehler BM. The Birds of Paradise. Oxford: Oxford University Press; 1998.

9 Scholes E. Structure and composition of the courtship phenotype in the bird of paradise Parotia lawesii (Aves: Paradisaeidae). Zoology 2008; 111: 260-278.

10 Laman T, Scholes E. Birds of Paradise: Revealing the World's Most Extraordinary Birds. Washington, DC: National Geographic Society; 2012.

11 Leertouwer HL, Wilts BD, Stavenga DG. Refractive index and dispersion of butterfly scale chitin and bird keratin measured by interference microscopy. Opt Express 2011; 19: 24061-24066

12 Stavenga DG, Leertouwer HL, Wilts BD. Quantifying the refractive index dispersion of a pigmented biological tissue using Jamin-Lebedeff interference microscopy. Light Sci App/ 2013; 2: e100; doi:10.1038/lsa.2013.56.

13 Stavenga DG, Leertouwer HL, Hariyama T, De Raedt HA, Wilts BD. Sexual dichromatism of the damselfly Calopteryx japonica caused by a melanin-chitin multilayer in the male wing veins. PIOS ONE 2012; 7: e49743.

14 Stavenga DG, Wilts BD, Leertouwer HL, Hariyama T. Polarized iridescence of the multilayered elytra of the Japanese jewel beetle, Chrysochroa fulgidissima. Philos Trans R Soc B 2011; 366: 709-723.

15 McGraw KJ. Mechanics of melanin-based coloration. In: Hill GE, McGraw KJ, editors. Bird Coloration. Vol. I, Mechanisms and Measurements. Cambridge, MA: Harvard University Press; 2006. p243-295.

16 Land MF. The physics and biology of animal reflectors. Progr Biophys Mol Biol 1972; 24: 75-106.

17 Brink DJ, van der Berg NG. Structural colours from the feathers of the bird Bostrychia hagedash. J Phys D: Appl Phys 2004; 37: 813-818.

18 Shawkey MD, Hauber ME, Estep LK, Hill GE. Evolutionary transitions and mechanisms of matte and iridescent plumage coloration in grackles and allies (Icteridae). J R Soc Interface 2006; 3: 777-786.

19 Stavenga DG. Thin film and multilayer optics cause structural colors of many insects and birds. Mater Today Proc 2014; 1S: 109-121.

(C) (i) (9) This work is licensed under a Creative Commons Attribution-

NonCommercial-ShareAlike 3.0 Unported License. The images or other third party material in this article are included in the article's Creative Commons license, unless indicated otherwise in the credit line; if the material is not included under the Creative Commons license, users will need to obtain permission from the license holder to reproduce the material. To view a copy of this license, visit http://creativecommons.org/licenses/by-nc-sa/3.0/ 